\title{
Modern management of arrhythmias
}

\author{
M C S Hall, D M Todd
}

Postgrad Med J 2006;82:117-125. doi: 10.1136/pgmj.2005.033654

The specialist management of arrhythmias has changed significantly over the past decade. This article outlines current management strategies for atrial flutter and atrial fibrillation, with particular emphasis on curative strategies with catheter ablation and the recent data on rhythm compared with rate control strategies. The expanding role of catheter ablation in the treatment of a wide variety of supraventricular and ventricular arrhythmias is discussed. The current evidence for implantable cardioverter defibrillators in the prevention of sudden cardiac death is summarised. The article also highlights the increasing recognition of a number of inherited syndromes that predispose to sudden cardiac death (for example, Brugada and long QT syndromes).

See end of article for authors' affiliations

......................

Correspondence to: Dr D M Todd, The Cardiothoracic Centre, Thomas Drive, Liverpool L14 3PE, UK; derick.todd@ ctc.nhs.uk

Submitted

14 February 2005

Accepted 13 June 2005
$\mathrm{T}$ he management of cardiac arrhythmias has changed tremendously over the past decade. During this time the two principal tools of modern arrhythmia management, catheter ablation and device therapy, have evolved to treat many conditions. Catheter ablation has moved patients with refractory arrhythmias to a first line treatment. In addition it has expanded into the treatment of the "big two" arrhythmiasatrial fibrillation $(\mathrm{AF})$ and atrial flutter. Device therapy has moved from bradycardia pacing into more complex areas such as the implantable cardiac defibrillator (ICD), which has been shown to be a very effective lifesaving device in patients at risk of or with a history of ventricular arrhythmias. Finally, cardiologists also have some answer to the growing tide of heart failure with biventricular pacing to permit cardiac resynchronisation.

The use of catheter ablation for patients with supraventricular tachycardia, especially those with Wolff-Parkinson-White (WPW) syndrome, has been an established therapy for several years. The principal advances in catheter ablation have been in the treatment of AF and flutter and also in the treatment of ventricular tachycardia (VT) (table 1). The advances in ablation therapy for these conditions have been greatly helped by the advent of modern non-fluoroscopic mapping systems that allow the operator to view cardiac anatomy, electrophysiology and catheter positions without the need for fluoroscopy.

There have been a number of important studies on the management of AF published in the past few years. These studies have focused on the role of rhythm management-that is, striving to maintain sinus rhythm, compared with from being a treatment considered only for acceptance of AF with appropriate control of the ventricular rate. We will discuss the findings and implications of these studies later.

Device therapy indications (table 2) have also changed considerably in the past few years. ICDs have become both smaller and more sophisticated, making a routine implant little different from implanting a permanent pacemaker. The advent of biventricular pacing has provided cardiologists with a genuine chance to improve the quality and quantity of life in patients with symptomatic heart failure.

\section{ABLATION FOR SUPRAVENTRICULAR ARRHYTHMIAS}

Although an established therapy for many years, improvements in electrophysiological techniques have led to catheter ablation becoming first line therapy for most patients with AV nodal reentrant tachycardia (AVNRT) or WPW, and for many patients with atrial flutter.

\section{AVNRT}

AVNRT is caused by re-entry within the AV node, using "fast" and "slow" pathways. Ablation of the slow pathway to cure AVNRT has become a comparatively straightforward and routine procedure. The most significant complication associated with slow pathway ablation is damage to the fast pathway and hence AV block requiring pacing. In one large series of more than 8000 patients, long term cure was achieved in $99 \%$ of patients with high grade AV block necessitating pacing occurring in only $0.4 \%{ }^{1}$ Catheter ablation has become the treatment of choice in patients with symptomatic AVNRT. Indeed, many patients and their physicians choose ablation as first line therapy to avoid the need for antiarrhythmic drug therapy.

\section{WPW syndrome}

Arrhythmias in the WPW syndrome are attributable to the presence of an additional atrioventricular connection, termed an "accessory pathway". This commonly presents with atrioventricular re-entrant tachycardia (AVRT), which is often well tolerated. However, patients with WPW are at risk should they develop atrial fibrillation as the accessory pathway can conduct more rapidly than the AV node. Very rapid
Abbreviations: AF, atrial fibrillation; $I C D$, implantable cardiac defibrillator; WPW, Wolff-Parkinson-White syndrome; VT, ventricular tachycardia; VF, ventricular fibrillation; AVNRT, AV nodal re-entrant tachycardia; AVRT, atrioventricular re-entrant tachycardia; CTI, cavotricuspid isthmus; TdP, torsade de pointes; LACA, left atrial cicumferential ablation; $\mathrm{PVI}$, pulmonary vein isolation; RFA, radiofrequency catheter ablation; LVEF, left ventricular ejection fraction; EP, electrophysiological study; SCD, sudden cardiac death 
Table 1 Indications for radiofrequency catheter ablation of cardiac arrhythmias

\begin{tabular}{lll}
\hline 1st line therapy & 2nd line after medical therapy & Occasionally useful \\
\hline Wolff-Parkinson-White syndrome & Atrial flutter & Chronic AF \\
$\begin{array}{l}\text { AV nodal re-entrant tachycardia } \\
\text { Atrial flutter (selected patients) }\end{array}$ & $\begin{array}{l}\text { Paroxysmal AF } \\
\text { "Normal heart" VT }\end{array}$ & $\begin{array}{l}\text { VT in the presence of structural heart } \\
\text { disease }\end{array}$ \\
\hline
\end{tabular}

ventricular rates and ventricular fibrillation may ensue.

RF ablation of accessory pathways has become first line therapy for patients with symptomatic WPW as antiarrhythmic drugs are often ineffective and poorly tolerated. In a study of over 6000 patients from several centres, long term success rates were $98 \%$. Serious complications (cardiac tamponade, AV block, coronary dissection, and stroke) were seen in $0.6 \%$ with only one fatality. ${ }^{1}$ The one time risk of catheter ablation seems to be lower than the cumulative lifetime risk associated with symptomatic WPW.

\section{Atrial flutter}

Atrial flutter is caused by a single wave of electrical activation that takes a consistent path around the atria. About $90 \%$ of atrial flutter involves a counterclockwise rotation in the right atrium. This pattern is often called "typical" or "common" atrial flutter by electrophysiologists. Typical atrial flutter results in a saw-tooth pattern in the inferior leads of the 12 lead ECG and a positive F wave in Vl (fig 1). The atrial rate is often around 300/min with a resulting ventricular rate of 150/ min. The circuit involved in typical flutter has been well defined and involves a narrow bridge of tissue (normally l$2 \mathrm{~cm}$ ) between the tricuspid valve and the inferior vena cava (IVC). This area is called the cavotricuspid isthmus (CTI). Drawing a line of ablation across this area effectively blocks the circuit for typical flutter.

Over the past 10 years the technique for flutter ablation has evolved and we now have well defined end points for the procedure. ${ }^{2-4}$ By achieving these end points long term success rates in prevention of recurrent atrial flutter are about $80 \%-$ $90 \%$. A prospective, randomised trial of medical treatment compared with catheter ablation as the first line therapy in atrial flutter has shown significantly better outcomes for patients treated by ablation. Patients treated by ablation had reduced atrial flutter, better wellbeing, and also a reduction in the onset of atrial fibrillation. ${ }^{5}$ The high success rate of ablation (with a low incidence of complications) suggests that increasing use should be made of ablation for this arrhythmia. Flutter ablation should be considered as first line therapy for three groups of patients: those with troublesome symptoms, those in whom ventricular rate control with drugs is suboptimal, and in those with left ventricular dysfunction as a result of persistent tachycardia.

The principal weakness of CTI ablation for atrial flutter is the subsequent onset of AF. Although atrial flutter and AF are different arrhythmias they are strongly connected. They share many predisposing factors and many patients will present with a combination of both atrial flutter and fibrillation. Patients with both arrhythmias who do especially well with flutter ablation are those in whom antiarrhythmic drug therapy (principally class Ic drugs such as flecainide) converts AF to flutter. ${ }^{6}$ After ablation the antiarrhythmic therapy must be continued to suppress further $\mathrm{AF}$, but arrhythmia control is generally good with this strategy. In some other patients atrial flutter is an important precursor to $\mathrm{AF}$ and flutter ablation can then also be an effective antiarrhythmic strategy.

\section{ATRIAL FIBRILLATION}

There are two important areas that have changed in the management of AF over the past five years. The first is the publication of several studies addressing the issue of rate compared with rhythm control. The second is the use of ablation as a curative therapy for patients with AF.

\section{Rate compared with rhythm control}

Much of the treatment of AF in the past 30 years has been based on the "best guess" principle. AF is associated with heart disease and patients with AF were known to have an increased risk of stroke ${ }^{7}$ and an increased mortality, ${ }^{8}$ and therefore it was considered logical to attempt to restore sinus rhythm. However, the pursuit of sinus rhythm was not necessarily free from risks. The most important of these risks was the well recognised side effect of proarrhythmia from antiarrhythmic drugs. ${ }^{9-11}$ This led several groups to ask whether a default strategy of rhythm control was correct for all patients with AF or whether certain groups may be better served by a policy of accepting AF and controlling the ventricular rate.

\begin{tabular}{ll} 
Table 2 Evidence based indications for ICD therapy \\
\hline $\begin{array}{l}\text { Primary prevention } \\
\text { (patients at risk of VT or VF) }\end{array}$ & $\begin{array}{l}\text { Secondary prevention } \\
\text { (patients resuscitated from VT or VF) }\end{array}$ \\
\hline $\begin{array}{l}\text { Patients with class II or III heart failure of any aetiology } \\
\text { with a LVEF }<35 \%\end{array}$ & $\begin{array}{l}\text { Cardiac arrest attributable to VT or VF not attributable } \\
\text { to a transient or reversible cause }\end{array}$ \\
$\begin{array}{l}\text { Patients with class III heart failure and a QRS duration } \\
\text { of }>120 \mathrm{~ms} \text { (these patients should be considered for a }\end{array}$ & \\
biventricular ICD) & \\
$\begin{array}{l}\text { Patients with LVEF }<30 \% \text { at least one month after MI or } \mathrm{MF} \text { with haemodynamic compromise } \\
3 \text { months after CABG }\end{array}$ & $\begin{array}{l}\text { Sustained VT or VF without haemodynamic } \\
\text { compromise but with LVEF }<35 \%\end{array}$ \\
$\begin{array}{l}\text { Non-sustained VT in patients with coronary disease, } \\
\text { previous MI, LVEF }<35 \% \text { and inducible VT at }\end{array}$ & \\
electrophysiological study \\
$\begin{array}{l}\text { Familial condition with risk of SCD } \\
\text { Brugada syndrome }\end{array}$ \\
Long QT syndrome \\
HOCM
\end{tabular}




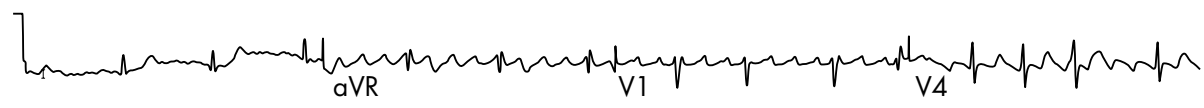

Figure 1 A 12 lead ECG of typical atrial flutter. Negative (saw-tooth) flutter waves are seen in the limb leads II, III, and aVF.
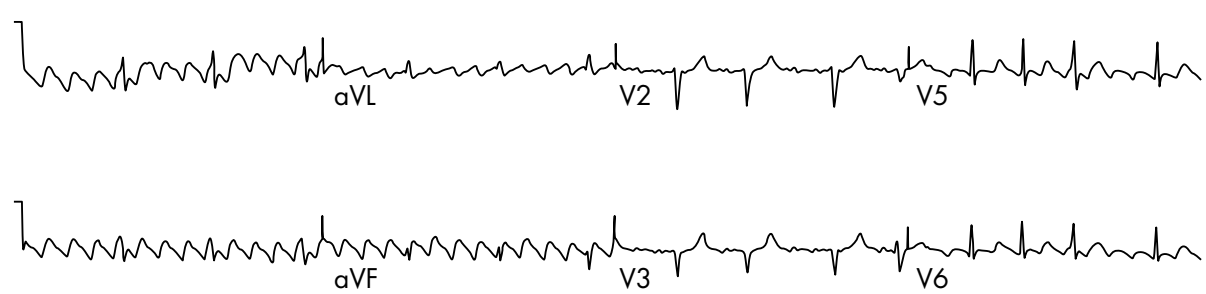

There have been four important studies of this aspect of AF management published in the recent few years. ${ }^{12-15}$ The largest of these studies was the AFFIRM study. ${ }^{14}$ Of the four studies, this study had the broadest inclusion criteria, and as treatment within each arm was at the discretion of the physician, the full range of treatments of AF. It is important to understand the inclusion criteria for each of the studies to allow us to focus on their applicability to the management of AF in clinical practice. For AFFIRM the inclusion criteria were patients aged $>65$ years, or with another risk factor for stroke or death, in whom the physician judged that AF would be an ongoing clinical problem. On this basis both patients with paroxysmal and persistent AF were enrolled. The other three studies only included patients with persistent AF. All patients had to be eligible for treatment with anticoagulants. Importantly, before randomisation the physician had to make a judgment that either treatment strategy would be acceptable to the patient. Many patients were enrolled during their first episode of AF (34\%). A total of 4060 patients were enrolled and followed up for a mean of 3.5 years. The cross section of patients enrolled was similar to that seen in clinical practice here in the UK. The results are summarised below:

(1) There was no difference in the primary outcomes of death and stroke during the time of follow up.

(2) There was a slight trend to more strokes in the rhythm control group, attributed to the tendency of physicians to withdraw anticoagulation when patients appeared to be stable in sinus rhythm. This provides an important lesson from this study. When a patient has an indication for anticoagulant treatment in the presence of AF even successful rhythm control does not remove the need for ongoing anticoagulation. A detailed review of indications for anticoagulation is beyond the scope of this article and the reader is referred to a recent review. ${ }^{16}$

(3) The main difference between the two treatment arms was that those in the rhythm control arm consumed more healthcare time and resources (for treatments such as DC cardioversion, pacemaker implantation, etc).

(4) The risk of serious ventricular arrhythmia (torsade de pointes) was low (12 of 2030 or $0.8 \%$ ) in the rhythm control arm, but still important.

The PIAF study ${ }^{15}$ compared rate control with diltiazem with rhythm control with amiodarone \pm DC cardioversion in 252 patients with persistent AF (duration 7 to 360 days). Amiodarone was effective at maintaining sinus rhythm in $56 \%$ of patients at one year, compared with only $10 \%$ in sinus rhythm in the diltiazem group. The primary end point was symptoms related to $\mathrm{AF}$ and was no difference between the groups, although the rhythm control group had a slightly better exercise tolerance on a six minute walk test.
The RACE study ${ }^{13}$ investigated patients with recurrent AF after DC cardioversion. Patients were randomised to a policy of rate control compared with a rhythm control policy consisting of serial DC cardioversions with ongoing antiarrhythmic drug treatment in an attempt to maintain sinus rhythm. A total of 522 patients were followed up for 2.3 years. The authors found no difference in a wide range of adverse events between the two groups.

The STAF study ${ }^{12}$ compared a strategy of rhythm control with rate control in patients with persistent AF. A total of 200 patients were recruited. There was no difference in the occurrence of major end points between the two arms of the study over three years. The authors did note, however, that most (18 of 19) of the serious end points occurred in AF.

As alluded to above, the STAF study ${ }^{12}$ and AFFIRM ${ }^{14}$ both showed an increased risk of serious adverse events for patients in $\mathrm{AF}$, or put another way, a reduction in adverse events for patients who remained in sinus rhythm. Cardioversion of AF and maintenance of sinus rhythm has also previously been shown to result in better outcomes in two studies of antiarrhythmic drugs in heart failure patients. ${ }^{17}{ }^{18}$ Studies of rate versus rhythm control in AF have failed to show an advantage to a rhythm control policy, despite the fact that patients in sinus rhythm do better than those who remain in $\mathrm{AF}$.

This paradox is explained by the fact that the survival advantage of sinus rhythm is negated by a survival disadvantage when taking antiarrhythmic drug therapy. Perhaps when we have an effective alternative to antiarrhythmic drug therapy for the maintenance of sinus rhythm then we should readdress the issue of rate compared with rhythm control. However, current advice must be that if AF is well tolerated then a strategy of ventricular rate control is quite acceptable. It should also be emphasised that once a strategy of rate control is applied all antiarrhythmic drug teatment should be withdrawn.

The situation is much more difficult in the symptomatic patient. In these patients one must attempt to reduce symptoms by restoring sinus rhythm, but we must be aware of the possibility of serious side effects from our treatments.

\section{Ablation for atrial fibrillation}

Research into effective treatments for AF has flourished over the past decade. Increased understanding of the electrophysiology of AF from experimental work combined with findings from the catheter laboratory has led to effective treatments for AF. Effective control of AF was only initially possible by open heart surgery and the Maze procedure. ${ }^{19}$ Performing a catheter based Maze procedure for the control of AF was unsuccessful but led to the finding that in a large proportion of patients AF was triggered by ectopy originating in the pulmonary veins. After this finding Haissaguerre and 
colleagues in Bordeaux went on to describe effective treatment of AF by radiofrequency ablation. ${ }^{20}$

Since the description of successful treatment of AF by catheter ablation, techniques have evolved considerably. Early enthusiasm was dented by the appreciation that extensive ablation in the left atrium was associated with a risk of stroke. Ablation within the pulmonary veins was also shown to result in pulmonary vein stenosis/occlusion. The target area for ablation has now moved to the atrial aspect of the pulmonary veins with improved results and reduced complications. The two principal techniques that have gained the most widespread acceptance are pulmonary vein isolation (PVI) as pioneered by the group in Bordeaux, ${ }^{21} 22$ and the left atrial circumferential ablation (LACA) technique as developed in Milan by Carlo Pappone. ${ }^{23}{ }^{24}$ A recent comparison of the two techniques by another group has suggested a slightly higher efficacy of LACA over PVI ${ }^{25}$ although the recent addition of linear ablation in the left atrium to PVI has further increased its effectiveness. These two pioneering groups are reporting cure rates of up to $90 \%$ for patients with paroxysmal AF and only slightly lower for patients with persistent AF. The Bordeaux group have shown that, in patients with heart failure and AF, PVI improves left ventricular function, heart failure symptoms, and quality of life. ${ }^{26}$ Pappone's group have shown improvements in all cause mortality, mainly attributable to a reduction in heart failure and ischaemic stroke, in those patients undergoing LACA compared with control. ${ }^{27}$ Although neither of these studies were randomised, they provide promising initial evidence that ablation for AF is safe and may have a beneficial effect on mortality. In this respect ablation seems to have an advantage over pharmacological rhythm control.

Outside of the pioneering centres, however, the results are not quite as good. Cure rates for paroxysmal AF are around $70 \%$, but for persistent $\mathrm{AF}$ are somewhat less than $50 \% .{ }^{28}$ Complication rates are also higher; a recent worldwide survey of complications after AF ablation showed an overall risk of significant complications of $6 \%$ including a $1 \%-2 \%$ risk of cardiac tamponade and a $1.3 \%$ risk of pulmonary vein stenosis. ${ }^{29}$ Although most complications can usually be managed successfully without serious long term sequelae, some of the rarer complications carry significant mortality, for example, atrio-oesophageal fistula.

Currently most interventional cardiac centres in the UK offer ablation for AF although patient selection and numbers vary considerably. Catheter ablation is certainly a treatment that should be considered for all patients who are symptomatic in $\mathrm{AF}$ and not adequately controlled on or are intolerant of antiarrhythmic medication.

\section{VENTRICULAR TACHYCARDIA AND VENTRICULAR FIBRILLATION}

Ventricular fibrillation (VF) and ventricular tachycardia (VT) are most commonly the consequence of coronary heart disease. $^{30}$ They differ fundamentally from supraventricular tachycardias in that they are a common cause of sudden cardiac death (SCD). Their current management has been affected by the following:

(1) The widespread adoption of implantable cardioverter defibrillators (ICDs) for first line prevention of VT and VF

(2) The recognition of specific syndromes, for example, Brugada, long QT, which are associated with a high incidence of arrhythmic death attributable to VT and VF

(3) The recognition that VT occurring in a structurally normal heart is potentially curable by radiofrequency catheter ablation (RFA), and that VT in the setting of structural heart disease is also amenable to effective treatment by RFA.

\section{Key points: supraventricular arrhythmias}

- Radiofrequency ablation is first line therapy for SVT

- Atrial flutter is curable by ablation in $90 \%$ of patients

- A rate control strategy with oral anticoagulation is acceptable for many patients with AF

- For patients with severe symptoms attributable to AF, ablation for AF is becoming more widely available

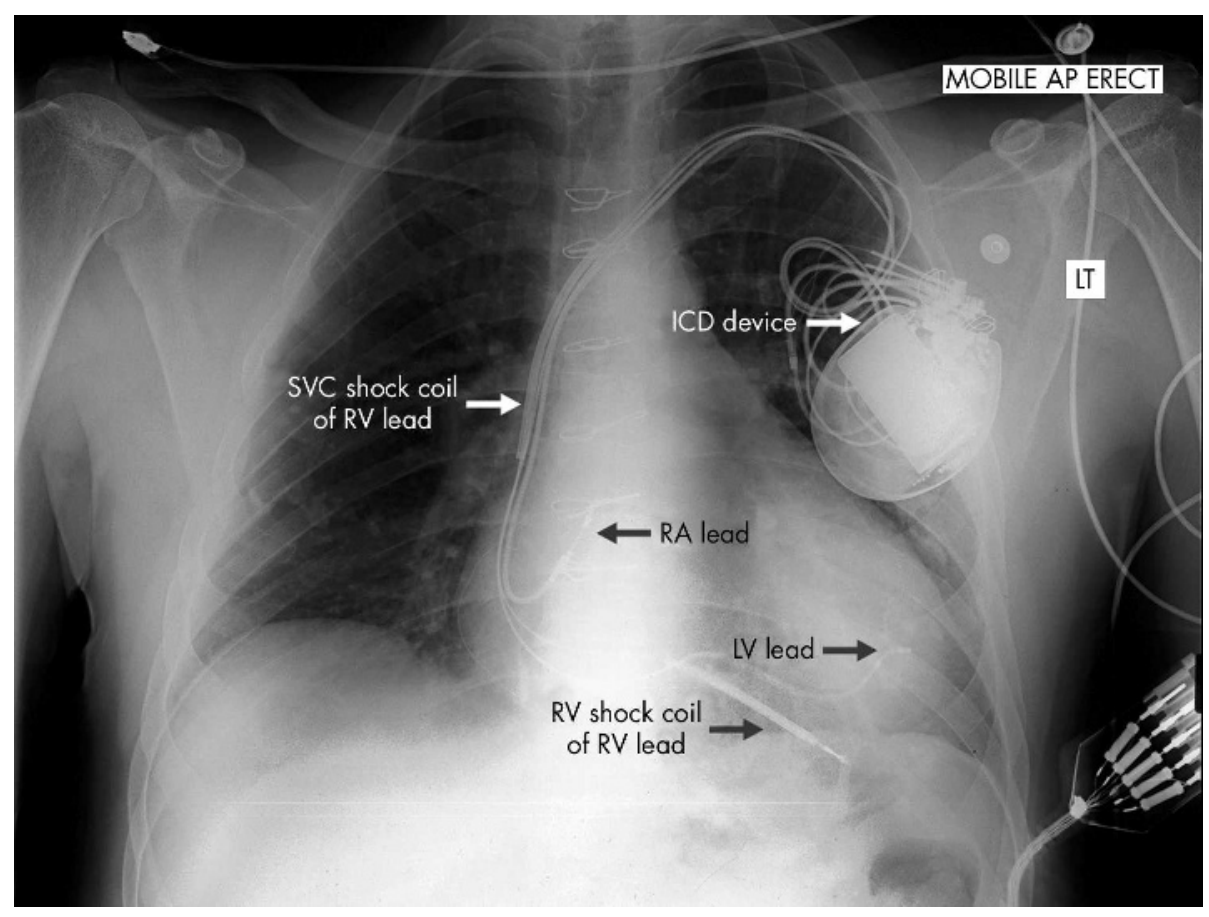

Figure 2 Chest radiograph showing a biventricular ICD in situ. Note the position of the device in the left pectoral region. A right ventricular lead with two shock coils, a right atrial lead, and a left ventricular lead (passed into an epicardial vein via the coronary sinus) are arrowed. 
This section will focus on the long term management of patients who have been successfully resuscitated from an episode of VT or VF (secondary prevention) and those considered to be at high risk of VT or VF (primary prevention). For the acute treatment of VT and VF (advanced life support, cardioversion and acute antiarrhythmic drug therapy) the reader is referred to the current European Resuscitation Council advanced life support guidelines. ${ }^{31}$

\section{ICD therapy for VT and VF}

The management of patients who have survived a cardiac arrest attributable to VT or VF by pharmacological means has generally been disappointing. ICDs became commercially available in the 1980s and early 1990s. Three prospective trials (AVID, ${ }^{32} \mathrm{CASH}^{33}$ and $\mathrm{CIDS}^{34}$ ) compared ICD therapy with antiarrhythmic drug therapy, predominantly with amiodarone, in survivors of life threatening ventricular arrhythmias. A meta-analysis of these studies showed a $25 \%$ reduction in all cause mortality, which was entirely attributable a $50 \%$ reduction in sudden death. ${ }^{35}$ A second meta-analysis showed that the effect was primarily limited to those patients with left ventricular ejection fraction (LVEF) $<35 \%{ }^{36}$

Together with the recognition of the efficacy of ICDs in patients surviving cardiac arrest, two clinical trials (MADIT $\mathrm{I}^{37}$ and MUSTT $^{38}$ ) have shown a survival benefit from prophylactic insertion of an ICD in patients at risk of serious ventricular arrhythmias. Patients at risk were identified as those with the combination of prior myocardial infarction, impaired left ventricular function, non-sustained (NS)VT on Holter monitoring, and inducible VT or VF at electrophysiological (EP) study.

A subsequent trial, MADIT II, ${ }^{39}$ showed a survival benefit from prophylactic ICD implantation in patients with a history of myocardial infarction and LVEF $<30 \%$, regardless of the presence of NSVT or the results of EP testing. Recently the SCD-HeFT trial has shown improved survival in NYHA class II and III patients with a LVEF $<35 \%$ receiving ICD implant regardless of the underlying aetiology of the left ventricular dysfunction. $^{40}$

Although ICDs prevent arrhythmic death, many patients receiving them have advanced coronary disease and/or significant left ventricular dysfunction. Patients who would previously have succumbed to an arrhythmic death may now find themselves in the advanced stages of heart failure. Biventricular pacing has been shown to significantly improve symptoms and exert a modest effect on prognosis in patients with severe left ventricular impairment attributable to any cause $(\mathrm{LVEF}<35 \%)$ and class III or IV heart failure. ${ }^{41}$ When considering ICD therapy for such patients it may be appropriate to implant a device capable of biventricular pacing.

Table 2 summarises current evidence based indications for ICD implantation. ${ }^{42}$ Not all countries have fully adopted all of these ICD indications in view of concerns regarding cost and the medical infrastructure to support implantation and follow up.

ICD implantation is usually a straightforward procedure not dissimilar to a pacemaker implant. It can usually be carried out under local anaesthesia and sedation. The ventricular lead differs from a conventional pacing lead in that it contains coils that permit a high voltage shock to be delivered without local damage to the myocardium. As part of the ICD implant procedure, VF is induced and the ability of the device to detect and treat it tested. The risk of ICD implant is low, with pneumothorax and infection the commonest complications. The chest radiograph in figure 2 shows the usual site and size of the ICD generator as well as the lead positions. In this case a biventricular device has been implanted and a left ventricular lead is visible.

Modern ICDs have advanced algorithms that help to discriminate ventricular arrhythmias. Nevertheless, occasionally shock therapy can be delivered inappropriately, for example, during supraventricular tachycardias (for example, $\mathrm{AF}$ ) or if a mechanical problem such as a lead fracture develops. Shock therapy is painful and may have profound psychological effects on the patient as well as having implications for social activities such as driving.

\section{Antiarrhythmic drugs to prevent VT and VF}

Antiarrhythmic drugs as a group have limited efficacy for preventing VT..$^{43}$ Their present role is as an adjunct to ICD implantation to reduce number of shocks or in patients who do not wish to have an ICD. When used, empiric amiodarone is usually the drug of choice..$^{43} 44$

A number of clinical trials have evaluated the efficacy of class Ia, Ib, Ic, and III drugs in the long term prevention of ventricular arrhythmias. None were placebo controlled for ethical reasons. A consistent message from these trials is that antiarrhythmic drugs may be pro-arrhythmic, especially in patients with coronary disease. Specifically, class Ic drugs, for example, flecainide, are contraindicated in patients with coronary disease. ${ }^{45}$ Other class I drugs are rarely used because of concerns about pro-arrhythmic effects.

The class III drugs amiodarone and sotalol are in any case more effective than class I drugs in the treatment of VT. ${ }^{43} 4647$ However, the negatively inotropic effects of sotalol limit its use in patients with left ventricular impairment: in these
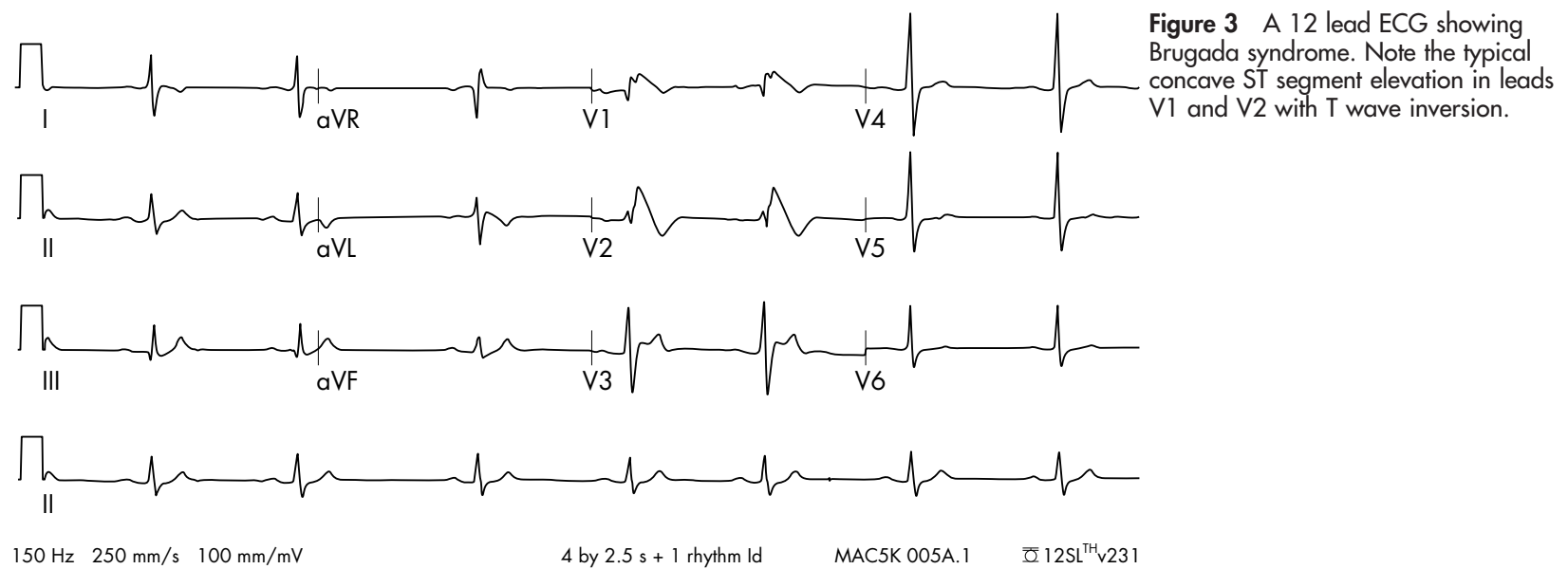
A

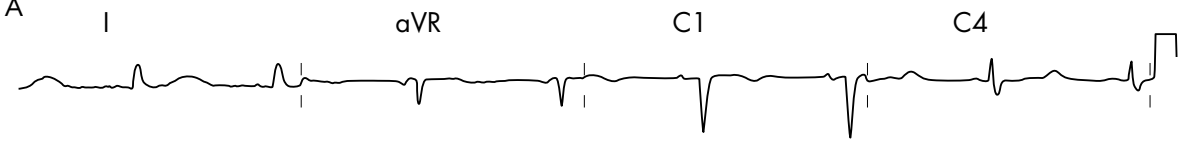

$m_{1}$

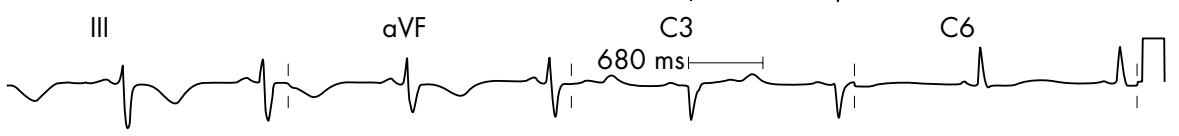

॥

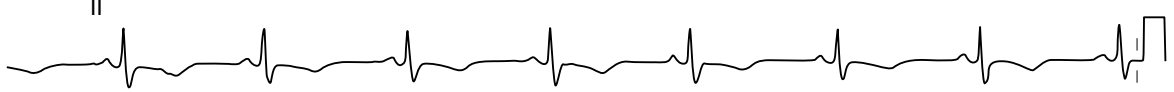

$25 \mathrm{~mm} / \mathrm{s} 10 \mathrm{~mm} / \mathrm{mV}$ F 0.5 Hz-40 Hz W HP709 07876

B

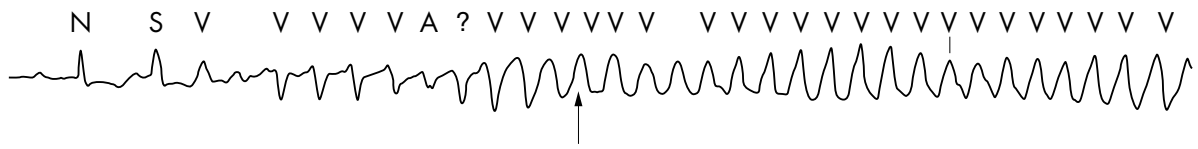

Figure 4 (A) A 12 lead ECG showing drug induced QT prolongation. The QT interval measured in lead V3 is $680 \mathrm{~ms}$. The $T$ wave morphology is also abnormal with $T$ wave inversion in leads II, III, and aVF and notched T waves in leads V2 and V3. (B) Rhythm strip showing onset of torsades de pointes ventricular tachycardia. Note the rotation of the VT with initially negative QRS complexes changing to positive QRS complexes at the point indicated by the arrow. patients amiodarone is the drug of choice. ${ }^{32}$ The long term efficacy of newer class III drugs such as dofetilide has not yet been fully evaluated. Initial studies suggest that it is as effective as sotalol and better tolerated. ${ }^{48}$

\section{VT and VF in the structurally normal heart}

Although the vast majority of cases of VT and VF occur in the setting of impaired left ventricular function, a number of conditions are recognised that cause these arrhythmias in the apparently structurally normal heart. It is important to recognise these arrhythmias as they may be precipitated by certain drugs, may be inherited, or may be amenable to catheter ablation.

\section{Brugada syndrome}

The Brugada syndrome was first described in $1992 .{ }^{49}$ It presents most often in young males, particularly in the Asian population. ${ }^{50}$ It is diagnosed by a typical ECG appearance consisting of partial right bundle branch block together with persistent ST elevation and inverted or bifid T waves in leads V1 to $\mathrm{V}^{49}$ (fig 3). Some patients do not have the typical ECG appearances at rest but they become apparent after the administration of a sodium channel blocking drug such as flecainide or ajmaline. ${ }^{51}$

Patients with Brugada syndrome are at risk of SCD because of VT or VF. ${ }^{52}$ SCD may be the presenting feature; alternatively patients can present with syncopal episodes. ${ }^{52}$ The condition is inherited in an autosomal dominant fashion with variable expression. As a result, a significant number of asymptomatic patients are diagnosed through family screening.

The exact pathophysiological mechanism of SCD in these patients is not precisely known, although a significant proportion have abnormalities of sodium channel function. ${ }^{53}$ It is possible that changes in autonomic tone predispose towards arrhythmias as arrhythmias are often nocturnal. ${ }^{54}$

Antiarrhythmic drug therapy such as amiodarone or $\beta$ block is ineffective at preventing SCD in Brugada syndrome. ${ }^{55}$ Sodium channel blocking drugs are pro-arrhythmic. ${ }^{51}$ ICD implantation is effective in selected patients. ${ }^{56}$ ICD implantation should be undertaken in patients with the Brugada syndrome who have survived SCD or who have syncope. ${ }^{56}$

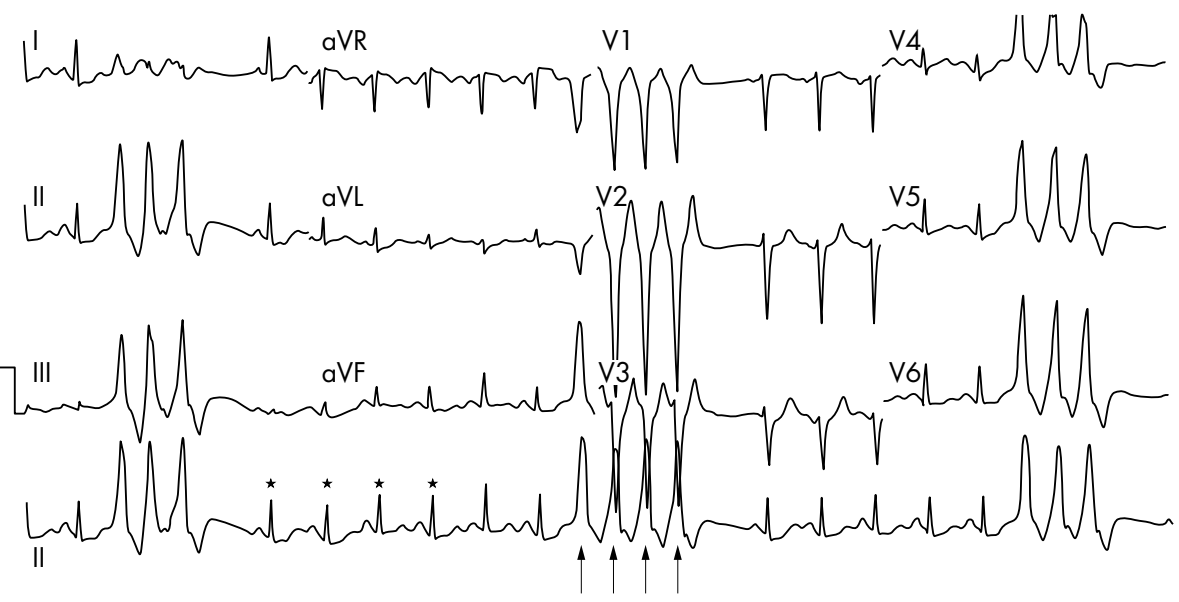

Figure 5 A 12 lead ECG showing bursts of three to four beats of right ventricular oufflow tract ventricular tachycardia (RVOT VT) interspersed with sinus rhythm. The sinus beats are associated with a normal QRS morphology (*). The RVOT VT ( $\uparrow$ ) has a typical left bundle branch block (LBBB) morphology in $\mathrm{V} 1$ with positive QRS in leads II, III, and aVF. 
(1)

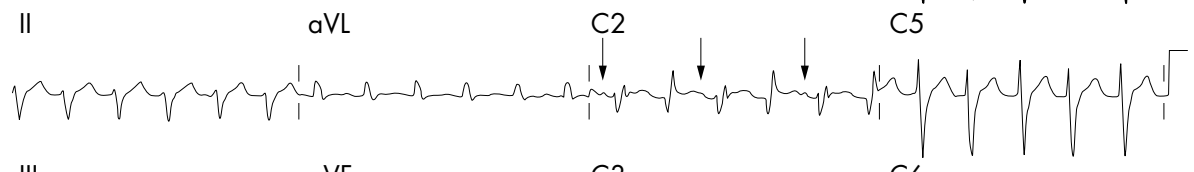

III

II

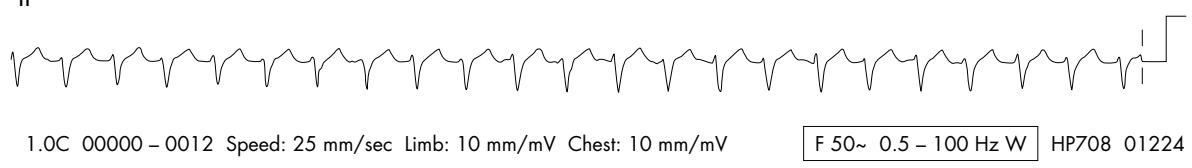

Figure 6 A 12 lead ECG showing idiopathic left ventricular (fascicular) VT. The VT has a right bundle branch block (RBBB), left axis pattern. Evidence of $A V$ dissociation is seen with $P$ waves in $\mathrm{V} 2(\downarrow)$. The $\mathrm{P}$ wave rate is almost exactly half the VT rate resulting in an apparent 2 to 1 pattern. The QRS following each $P$ wave has a slight change in morphology indicating that these are fusion beats.
There is still debate as to whether to implant ICDs in asymptomatic people with ECG changes characteristic of the syndrome. Electrophysiological testing has been advocated by some authors as useful in risk stratification, but more information is needed before this can be routinely recommended.

The presence of Brugada syndrome should be considered in any patient who has survived SCD and who has an apparently structurally normal heart. A normal resting ECG does not exclude the condition and these patients should have a challenge with a class Ic antiarrhythmic drug.

\section{Long QT syndrome}

The long QT syndrome represents a group of disorders of ventricular repolarisation, typified by prolongation of the QT interval on the ECG (fig 4A). The normal corrected QT interval (QTc) is less than $440 \mathrm{~ms}$. Although by definition in patients with LQTS the QTc is usually $>440 \mathrm{~ms}$, it may exhibit variation and be normal at times. A single normal recorded QTc does not, therefore, exclude the diagnosis. ${ }^{57}$ LQTS is associated with an increased risk of a life threatening form of ventricular arrhythmia called torsade de pointes (TdP) (fig 4B). The longer the QT interval, the higher the risk of TdP. ${ }^{58}$

LQTS is usually an inherited condition. Seven defects, predominantly in genes coding for cardiac ion channel proteins, have been identified and termed LQT1 to LQT7. The first three (LQT1, LQT2, and LQT3) are the commonest and most clinically relevant. ${ }^{59}$ Two clinically defined syndromes exist. The Romano-Ward syndrome is an autosomal dominant condition with no extracardiac manifestations. It may be caused by any of the underlying genetic mutations. ${ }^{60}$ The Jervell and Lange-Neilsen syndrome is autosomal recessive and associated with sensorineural deafness. It is only associated with mutations LQT1 and LQT5. ${ }^{60}$

Patients often present with a cardiac event, usually syncope, aborted cardiac arrest, or SCD. ${ }^{61}$ The condition is more apparent clinically in women, particularly when drug induced. ${ }^{62}$ A significant number of cases are diagnosed by screening of family members.

The risk of SCD seems to be highest in those who have already experienced symptoms or who have reported ventricular arrhythmias. Events are frequently triggered by exercise, auditory stimuli, or emotion. ${ }^{63}$

Assessing risk in apparently asymptomatic people, for example family members, is much more difficult. Current risk stratification is based on the genotype, the QT interval, and the sex of the patient. ${ }^{62}$ Forms associated with deafness and with the LQT3 variant seem to be at particularly high risk. $^{62}$

LQTS may also be acquired. Metabolic abnormalities, principally hypokalaemia and hypomagnesaemia, bradycardia of any cause, and a large number of drugs have been implicated. The distinction between acquired and inherited LQTS is made more complicated by the variable penetrance exhibited by many of the genetic forms. Many patients appear to have a "forme fruste" of LQTS, which becomes clinically apparent only when the patient is exposed to a second factor, for example a drug that prolongs the QT interval. The ongoing drug induced arrhythmia risk evaluation (DARE) study has been designed to explore the interaction between genetic and environmental factors in drug induced ventricular arrhythmias.

Treatment in acquired LQTS consists first of removing the stimulus and correcting any metabolic/electrolyte abnormalities or bradycardia. Antiarrhythmic drugs must be avoided but $\beta$ blockers, magnesium infusion, temporary pacing to prevent bradycardia, and phenytoin may be helpful in the acute setting. Patients with congenital LQTS need to avoid drugs that may precipitate TdP. A comprehensive, regularly updated list of such drugs can be found at http:// www.qtdrugs.org. Patients should be instructed to check all new prescribed drugs at this site.

The mainstay of preventative treatment is $\beta$ block, as sympathetic input is thought to be central to the initiation of arrhythmias. ${ }^{64}$ All patients with congenital LQTS, whether symptomatic or asymptomatic should be treated with a $\beta$ blocker in the absence of a contraindication. ${ }^{64}{ }^{65}$ About one third of patients with syncope or aborted SCD before $\beta$ blocker therapy will experience another cardiac event during a five year period taking $\beta$ blockers. ${ }^{62}$ Atrial pacing may also prevent further cardiac events. ${ }^{65}$ In patients presenting with

\section{Key points: ventricular arrhythmias}

ICD implantation is first line therapy for prevention of VT and VF

- Drug therapy alone for VT is usually insufficient

- "Normal heart" VT is often amenable to catheter ablation

- There is an increasing recognition of inherited syndromes, for example, LQTS, Brugada that predispose to arrhythmic death in young people 


\section{Five key references}

AFFIRM study

- Wyse DG, Waldo A, DiMarco JP, et al. A comparison of rate control and rhythm control in patients with atrial fibrillation. N Engl J Med 2002;347:1825-33.

\section{Catheter ablation for atrial fibrillation}

- Shah DC, Haissaguerre M, Jais P. Current perspectives on curative catheter ablation of atrial fibrillation. Heart 2002;87:6-8.

The use of ICDs to prevent sudden cardiac death

- Bardy GH, Lee KL, Mark DB, et al. Amiodarone or an implantable cardioverter-defibrillator for congestive heart failure. N Engl J Med 2005;253:225-37.

\section{Brugada syndrome}

- Antzelevitch C, Brugada P, Borggrefe M, et al. Brugada syndrome: report of the second consensus conference. Circulation 2005; 111:659-70.

\section{Long QT syndrome}

- Priori SG, Schwartz PJ, Napolitano C, et al. Risk stratification in the long-QT syndrome. $N$ Engl J Med 2003;348:1866-74.

cardiac arrest, the rate of repeat cardiac arrest is about $14 \%$ over a five year period of follow up and on this basis ICD implantation is recommended for all patients with LQTS who survive a cardiac arrest. ${ }^{62}$

All patients with a prolonged QTC on ECG or who present with TdP arrhythmias should be referred to a cardiac electrophysiologist for further investigation and screening of family members.

\section{"Normal heart" VT}

Ventricular tachycardia occurring in the presence of a structurally normal heart has two typical presentations. The first arises in the right ventricular outflow tract (RVOT), where it gives rise to a tachycardia with a typical left bundle branch block with strongly positive QRS in the inferior leads (fig 5). ${ }^{66}$ An important differential diagnosis for RVOT tachycardia is arrhythmogenic right ventricular dysplasia (ARVD). ARVD causes ventricular arrhythmias with similar ECG appearances to RVOT tachycardia but has a more sinister prognosis because of a coexisting cardiomyopathy ${ }^{67}$ Patients with ARVD often show resting abnormalities on the 12 lead ECG including $\mathrm{T}$ wave inversion in $\mathrm{Vl}-\mathrm{V} 3$, and right ventricular dilatation on echocardiography ${ }^{67}$

Idiopathic left ventricular tachycardia ${ }^{68}$ (fascicular VT) involves the posterior fascicle of the left ventricle and presents with a right bundle branch block, left axis deviation pattern (fig 6). It is amenable to radiofrequency ablation and is sensitive to verapamil. We do strongly recommend, however, that verapamil is never given to any patient with a broad complex tachycardia unless on the advice of a specialist electrophysiologist.

\section{CATHETER ABLATION FOR VT}

As discussed above catheter ablation is successful for VT in structurally normal hearts. RFA treatment of idiopathic VT arising from the RVOT has success rates between $75 \%$ and $100 \%,{ }^{66} 67{ }^{69}$ and for idiopathic left ventricular (fascicular) VT success varies from $50 \%$ to $90 \%{ }^{68}{ }^{70}$ In these arrhythmias RFA is the treatment of choice.
With the advent of non-fluoroscopic mapping systems more advanced forms of ablation are possible in patients with structural heart disease. This is important as the vast majority of patients have VT in relation to ischaemic heart disease. RFA may ablate selected VT morphologies with success rates of around 50\%. ${ }^{71}$ RFA has traditionally required that ablation be carried out during tachycardia and thus has not been an option in very rapid or poorly tolerated VT. However, newer "substrate mapping" techniques may permit ablation of such tachycardias during sinus rhythm or after delineation of potentially critical sites in the circuit by detailed mapping. ${ }^{73}$

After ablation, a significant proportion of patients still have inducible VT that may recur clinically ${ }^{74}$; these patients still require ICD implantation. Presently, RFA in ischaemic VT is used in patients who have ICDs in situ but who continue to experience VT despite antiarrhythmic therapy and ATP, requiring frequent shock therapy. It may also be used in patients who have well tolerated VT despite antiarrhythmic therapy in the presence of normal or only mildly impaired left ventricular function.

\section{CONCLUSION}

The specialist management of arrhythmias has changed significantly over the past decade. This is partly because of a significant increase in the evidence base, principally in the rate compared with rhythm debate for the management of atrial fibrillation. The recognition of Brugada syndrome and further data to permit risk stratification of the long QT syndrome have also been invaluable. In addition, as technology has advanced ablation has found a significant role in the management of all arrhythmias.

\section{Authors' affiliations}

M C S Hall, Manchester Heart Centre, Manchester, UK

D M Todd, The Cardiothoracic Centre, Liverpool, UK

Funding: none.

Conflicts of interest: none declared.

\section{REFERENCES}

1 Morady F. Catheter ablation of supraventricular arrhythmias: state of the art. Pacing Clin Electrophysiol 2004;27:125-42.

2 Shah DC, Haissaguerre M, Takahashi A, et al. Differential pacing for distinguishing block from persistent conduction through an ablation line. Circulation 2000;102:1517-22.

3 Shah DC, Takahashi A, Hocini M, et al. Local electrogram-based criteria of cavotricuspid isthmus block. J Cardiovasc Electrophysiol 1999;10:662-9.

4 Cauchemez B, Haissaguerre M, Fischer B, et al. Electrophysiological effects of catheter ablation of inferior cava- tricuspid annulus isthmus in common atrial flutter. Circulation 1996:93:284-94.

5 Natale A, Newby KH, Pisano E, et al. Prospective randomized comparison of antiarrhythmic therapy versus radiofrequency ablation in patients with atrial flutter. J Am Coll Cardiol 2000;35:1898-904.

6 Nabar A, Rodriguez LM, Timmermans $C$ et al. Effect of right atrial isthmus ablation on the occurrence of atrial fibrillation: observations in four patients having type I atrial flutter with or without associated atrial fibrillation. Circulation 1999;99:1441-5.

7 Wolf PA, Abbott RD, Kannel WB. Atrial fibrillation: a major contributor to stroke in the elderly. The Framingham study. Arch Intern Med 1987; 145:1561-4

8 Wolf P, Mitchell J, Baker C, et al. Impact of atrial fibrillation on mortality, stroke and medical costs. Arch Intern Med 1998;158:229-34.

9 Reimold SC, Cantillon CO, Friedman PL, et al. Propafenone versus sotalol for suppression of recurrent symptomatic atrial fibrillation. Am J Cardio 1993;71:558-63.

10 Coplen SE, Antman EM, Berline JA, et al. Efficacy and safety of quinidine therapy for maintenance of sinus rhythm after cardioversion. A meta-analysis of randomized control trials. Circulation 1990;82:1 106-16.

11 Flaker GC, Blackshear JL, Kronmal RA, et al. Antiarrhythmic drug therapy and cardiac mortality in atrial fibrillation. The Stroke Prevention in Atrial Fibrillation Investigators. J Am Coll Cardiol 1992;20:527-32.

12 Carlsson J, Miketic S, Windeler J, et al. Randomized trial of rate-control versus rhythm control in persistent atrial fibrillation: the strategies of treatment of atrial fibrillation (STAF) study. J Am Coll Cardiol 2003;41:1690-6. 
13 Van Gelder IC, Hagens VE, Bosker HA, et al. A comparison of rate control and rhythm control in patients with recurrent persistent atrial fibrillation. N Engl J Med 2002;347:1834-40.

14 Wyse DG, Waldo A, DiMarco JP, et al. A comparison of rate control and rhythm control in patients with atrial fibrillation. N Engl J Med 2002; 347: 1825-33.

15 Hohnloser SH, Kuck KH. Randomized trial of rhythm or rate control in atrial fibrillation: the pharmacological intervention in atrial fibrillation trial (PIAF). Eur Heart J 2001;22:801-2.

16 Crystal E, Connolly SJ. Role of oral anticoagulation in management of atrial fibrillation. Heart 2004;90:813-17

17 Pedersen OD, Bagger $\mathrm{H}$, Keller N, et al. Efficacy of dofetilide in the treatment of atrial fibrillation-flutter in patients with reduced left ventricular function. Circulation 2001; 104:292-6.

18 Deedwania PC, Singh BN, Ellenbogen K, et al. Spontaneous conversion and maintenance of sinus rhythm by amiodarone in patients with heart failure and atrial fibrillation. Circulation 1998;104:292-6.

19 Cox JL, Schuessler RB, Lappas DG, et al. An 8 1/2- year clinical experience with surgery for atrial fibrillation. Ann Surg 1996;224:267-73.

20 Haissaguerre $M$, Jais $P$, Shah DC, et al. Spontaneous initiation of atrial fibrillation by ectopic beats originating in the pulmonary veins. N Engl J Med 1998;339:659-66.

21 Shah DC, Haissaguerre $M$, Jais P. Current perspectives on curative catheter ablation of atrial fibrillation. Heart 2002;87:6-8

22 Shah DC, Haissaguerre $M$, Jais $P$, et al. Electrophysiologically guided ablation of the pulmonary veins for the curative treatment of atrial fibrillation. Ann Med 2000;32:408-16.

23 Pappone C, Oreto G, Rosiano S, et al. Atrial electroanatomic remodeling after circumferential radiofrequency pulmonary vein ablation: efficacy of an anatomic approach in a large cohort of patients with atrial fibrillation. Circulation 2001; 104:2539-44.

24 Pappone C, Rosiano S, Oreto G, et al. Circumferential radiofrequency ablation of pulmonary vein ostia: a new anatomic approach for curing atrial fibrillation. Circulation 2000;102:2619-28.

25 Oral H, Scharf C, Chugh A, et al. Catheter ablation for paroxysmal atrial fibrillation: segmental pulmonary vein ostial ablation versus left atrial ablation. Circulation 2003; 108:2355-60.

26 Hsu LF, Jais $P$, Sanders $P$, et al. Catheter ablation for atrial fibrillation in congestive heart failure. N Engl J Med 2004;351:2373-83.

27 Pappone C, Rosanio S, Augello G, et al. Mortality, morbidity and quality of life after circumferential pulmonary vein ablation for atrial fibrillation: outcomes from a controlled nonrandomized long-term study. J Am Coll Cardiol 2003;42:185-97.

28 Bourke JP, Dunuwille A, O'Donnell D, et al. Pulmonary vein ablation for idiopathic atrial fibrillation: six month outcome of first procedure in 100 consecutive patients. Heart 2005;91:51-7.

29 Cappato R, Calkins H, Chen SA, et al. Worldwide survey on the methods, efficacy and safety of catheter ablation for human atrial fibrillation. Circulation 2005;111:1100-5.

30 Demirovic J, Myerburg RJ. Epidemiology of sudden cardiac death: an overview. Prog Cardiovasc Dis 1994;37:39-48.

31 Latorre Fd, Nolan J, Robertson C, et al. European Resuscitation Council Guidelines 2000 for advanced adult life support. Resuscitation 2001;48:211-21

32 The AVID investigators. A comparison of anti-arrhythmic drug therapy with implantable defibrillators in patients resuscitated from near-fatal ventricular arrhythmias. N Engl J Med 1997;337:1576-84.

33 Kuck KH, Cappato R, Siebels J, et al. Randomized comparison of antiarrhythmic drug therapy with implantable defibrillators in patients resuscitated from cardaic arrest. The cardiac arrest study Hamburg (CASH) Circulation 2000;102:748-54.

34 Connolly SJ, Gent M, Roberts RS, et al. Canadian implantable defibrillator study (CIDS): a randomized trial of the implantable cardioverter defibrillator against amiodarone. Circulation 2000;101:1297-302.

35 Lee DS, Green LD, Liu PP, et al. Effectiveness of implantable defibrillators for preventing arrhythmic events and death: a meta analysis. J Am Coll Cardiol 2003;41:1573-82

36 Connolly SJ, Hallstrom AP, Cappato R, et al. Meta-analysis of the implantable cardioverter defibrillator secondary prevention trials. AVID, CASH and CIDS studies. Eur Heart J 2000;21:2071-8.

37 Moss AJ, Hall WJ, Cannom DS, et al. Improved survival with an implanted defibrillator in patients with coronary disease at high risk for ventricular arrhythmia. Multicenter Automatic Defibrillator Implantation Trial Investigators. N Engl J Med 1996;335:1933-40.

38 Buxton $\mathrm{AE}$, Lee $\mathrm{KL}$, Fisher JD, et al. A randomized study of the prevention of sudden death in patients with coronary artery disease. Multicenter Unsustained Tachycardia Trial Investigators. N Engl J Med 1999;341:1882-90.

39 Moss AJ, Zareba W, Hall WJ, et al. Prophylactic implantation of a defibrillator in patients with myocardial infarction and reduced ejection fraction. N Engl J Med 2002;346:877-83.

40 Bardy GH, Lee KL, Mark DB, et al. Amiodarone or an implantable cardioverter-defibrillator for congestive heart failure. N Engl J Med 2005;253:225-37.

41 Bristow MR, Saxon LA, Boehmer J, et al. Cardiac-resynchronization therapy with or without an implantable defibrillator in advanced chronic heart failure. N Engl J Med 2004;350:2140-50.

42 Gregoratos G, Abrams J, Epstein AE, et al. ACC/AHA/NASPE guideline update for implantation of cardiac pacemakers and antiarrhythmia devices. J Cardiovasc Electrophysiol 2002;13:1183-99.
43 The CASCADE investigators. Randomised antiarrhythmic therapy in survivors of cardiac arrest (the CASCADE study). Am J Cardiol 1993;72:280.

44 Mason JW. A comparison of electrophysiologic testing with Holter monitoring to predict antiarrhythmic drug efficacy for ventricular arrhythmias. N Engl J Med 1993:329:445-51.

45 Echt DS, Liebson PR, Mitchell LB. Mortality and morbidity in patients receiving encainide, flecainide, or placebo. The cardiac arrhythmia suppression trial. N Engl J Med 1991;324:781-8.

46 Haverkamp W, Matinez-Rubio A, Hief C, et al. Efficacy and safety of d, Isotalol in patients with ventricular tachycardia and in survivors of cardiac arrest. J Am Coll Cardiol 1997;30:487-95.

$47 \mathrm{Sim}$ I, McDonald KM, Lavori PW, et al. Quantative overview of randomized trials of amiodarone to prevent sudden cardiac death. Circulation 1997;96:2823-9.

48 Boriani G, Lubinski A, Capucci A, et al. A multicentre, double-blind randomized crossover comparative study on the efficacy and safety of dofetilide vs sotalol in patients with inducible sustained ventricular tachycardia and ischaemic heart disease. Eur Heart J 2001;22:2180-91.

49 Brugada P, Brugada J. Right bundle branch block, persistent ST elevation and sudden cardiac death: a distinct clinical and electrocardiographic syndrome. A multicenter report. J Am Coll Cardiol 1992;20:1391-6.

50 Alings M, Wilde A. A "Brugada" syndrome: Clinical data and suggested pathophysiological mechanism. Circulation 1999;99:666-73.

51 Brugada R, Brugada J, Antzelevitch C, et al. Sodium channel blockers identify risk for sudden death in patients with ST elevation and right bundle branch block but structurally normal hearts. Circulation 2000;101:510-15.

52 Brugada J, Brugada R, Antzelevitch C, et al. Long-term follow up of individuals with the electrocardiographic pattern of right bundle branch block and ST-segment elevation in precordial leads V1-V3. Circulation 2002; 105:73-8.

53 Priori SG, Napolitano C, Gasparini M, et al. Natural history of Brugada syndrome: insights for risk stratification and management. Circulation 2002; 105: 1342-7.

54 Matsuo K, Kurita M, Inagaki $M$, et al. The circadian pattern to the development of ventricular fibrillation in patients with Brugada syndrome. Eur Heart J 1999;20:465-70.

55 Brugada J, Brugada R, Brugada P. Right bundle branch block and ST-segment elevation in leads V1 through V3: a marker for sudden death in patients without demonstrable structural heart disease. Circulation 1998;97:457-60.

56 Priori SG, Aliot E, Blomstrom- Lundqvist C, et al. Task force on sudden cardiac death of the European Society of Cardiology. Eur Heart $J$ 2001;22:1374-450.

57 Moss AJ. Long QT syndrome. JAMA 2003;289:2041-4

58 Zareba W, Moss AJ, le Cessie A, et al. Risk of cardiac events in family members of patients with long QT syndrome. J Am Coll Cardiol 1995;26:1685-91.

59 Priori SG, Rivolta I, Napolitano C. Genetics of long QT, Brugada and other channelopathies. In: Zipes DP, Jalife J, eds. Cardiac electrophysiology:from cell to bedside.4th ed. Philadelphia: Saunders, 2004:462-70.

60 Chiang CE, Roden DM. The long QT syndromes: genetic basis and clinical implications. J Am Coll Cardiol 2000;36:1-12.

61 Locati EH, Zareba W, Moss AJ, et al. Age and sex-related differences in clinical manifestations in patients with congenital long QT syndrome. Circulation 1998;97:2237-44

62 Priori SG, Schwartz PJ, Napolitano C, et al. Risk stratification in the long-QT syndrome. N Engl J Med 2003;348:1866-74.

63 Moss AJ, Robinson JL, Gessmann L, et al. Comparison of clinical and genetic variables of cardiac events associates with loud noise versus swimming among subjects with the long QT syndrome. Am J Cardiol 1999;84:876-9.

64 Moss AJ, Zareba W, Hall WJ, et al. Effectiveness and limitations of beta blocker therapy in congenital long QT syndrome. Circulation 2000;101:616-23.

65 Dorostkar PC, Eldar M, Belhassen B, et al. Long term follow up of patients with long QT syndrome treated with beta blockers and continuous pacing. Circulation 1999; 100:2431-6.

66 Wilber DJ, Baerman J, Olshansky B, et al. Adenosine sensitive ventricular tachycardia: clinical characteristics and response to catheter ablation. Circulation 1993;87:126-34.

67 O'Donnell D, Cox D, Bourke JP, et al. Clinical and electrophysiological differences between patients with arrhythmogenic right ventricular dysplasia and right ventricular outflow tract tachycardia. Eur Heart J 2003;24:801-10.

68 Nakagawa H, Beckmann KJ, McClelland JH, et al. Radiofrequency ablation of idiopathic left ventricular tachycardia guided by a Purkinje potential. Circulation 1993;88:2607.

69 Stevenson WG, Nademanee K, Weiss JN, et al. Treatment of catecholaminesensitive right ventricular tachycardia by catheter ablation. J Am Coll Cardiol 1990;16:752.

70 Wellens HJ, Smeets JL. Idiopathic left ventricular tachycardia: cure by radiofrequency ablation. Circulation 1993;88:2978.

71 Schilling RJ, Peters NS, Davies DW. Feasibility of a noncontact catheter for endocardial mapping of ventricular tachycardia. Circulation 1999;99:2543-52

72 O'Donnell D, Bourke JP, Anilkumar R, et al. Radiofrequency ablation for post infarction ventricular tachycardia. Eur Heart J 2002;23:1699-705.

73 Arenal A, Glez-Torrecilla E, Ortiz M, et al. Ablation of electrograms with an isolated, delayed component as treatment of unmappable monomorphic ventricular tachycardias in patients with structural heart disease. J Am Coll Cardiol 2003;41:81-92.

74 Stevenson WG, Friedman PL, Kocovic D, et al. Radiofrequency catheter ablation of ventricular tachycardia after myocardial infarction. Circulation 1998;98:308. 